\title{
Análise cicloestratigráfica preliminar do sítio U1463C - Expedição 356 (IODP)
}

\author{
Muniz, Ana Luiza1,; Oliveira, R.R. ${ }^{1,2}$; Peixoto, I.M.C. ${ }^{1,2}$; Franco, Daniel R. ${ }^{2}$ \\ ${ }^{1}$ Departamento de Geologia e Geofísica, Instituto de Geociências, Universidade Federal Fluminense (IGc-UFF). \\ 2 Coordenação de Geofísica, Observatório Nacional (COGEO-ON/MCTIC).
}

\section{Copyright 2019, SBGf - Sociedade Brasileira de Geofísica}

This paper was prepared for presentation during the $16^{\text {th }}$ International Congress of the Brazilian Geophysical Society held in Rio de Janeiro, Brazil, 19-22 August 2019.

Contents of this paper were reviewed by the Technical Committee of the $16^{\text {th }}$ International Congress of the Brazilian Geophysical Society and do not necessarily represent any position of the SBGf, its officers or members. Electronic reproduction or storage of any part of this paper for commercial purposes without the written consent of the Brazilian Geophysical Society is prohibited.

\begin{abstract}
The northwest shelf (NWS) of Australia has become an important target of paleoceanographic studies due to its direct influence on fundamental processes of global ocean circulation, as well as to provide a reasonable stratigraphic preservation of important paleoenvironmental since the Pliocene. In view of the importance of this area of study, the following project aims to understand the paleoceanographic and geological dynamics through the investigation of the quasiperiodicities record in series of paleoclimatic proxies obtained along the stratigraphy of the International Ocean Discovery Program (IODP) Expedition 356 site U1463 $\left(18,97 \circ S ; 117,62^{\circ} E\right)$. In this study, spectral analysis results analysis based on the multitaper method (MTM) and and evolutionary harmonic analysis (EHA) will be presented from gamma ray and magnetic susceptibility series data obtained during during Expedition 356 of the IODP.
\end{abstract}

\section{Resumo}

A Plataforma Noroeste da Austrália (NWS, sigla em inglês) vem se tornando um dos principais alvos de estudos paleoceanográficos devido à influência direta com processos fundamentais de circulação oceânica global e por prover uma razoável preservação estratigráfica de importantes eventos paleoambientais desde o Plioceno. Com o objetivo compreender a dinâmica paleoceanográfica e geológica desta importante área de estudo, este trabalho investiga possíveis registros de quase-periodicidades em séries de proxies paleoclimáticos, obtidos ao longo da estratigrafia de um dos sete sítios de amostragem (sítio U1463 - 18, 97॰ S; 117, 62。 E) da Expedição 356 do International Ocean Discovery Program (IODP - sigla em inglês). Neste trabalho, serão apresentados resultados de análise espectral baseada no método multitaper (MTM- sigla em inglês) e análise harmônica evolutiva (EHA- sigla em inglês) em séries de dados de raios gama e susceptibilidade magnética, obtidos durante a Expedição 356 do IODP

\section{Introdução}

Segundo diversos autores (Veevers, Powell e Roots 1991, Gallagher et al. 2009, Gallagher et al. 2012), a sedimentação da bacia de margem passiva do noroeste da Austrália resume a história geológica da margem norte do país desde o Mioceno Superior até os dias atuais. A NWS, região de estudo, oferece condições singulares para a compreensão de elementos-chave da circulação termohalina global, devido à sua proximidade da chamada "Piscina Aquecida do Indo-Pacífico" (IPWP, sigla em inglês) e Fluxo Indonésio de Correntes (ITF, sigla em inglês; Fig. 1), importantes para o sistema climático global. A IPWP opera como uma espécie de "interruptor" do sistema climático global, e está sujeita à variabilidade em escala decadal devido ao El Niño/Oscilação Sul (ENSO) (Garidel-Thoron et al. 2005) exercendo, assim, importante influência na mudança climática global a curto prazo.

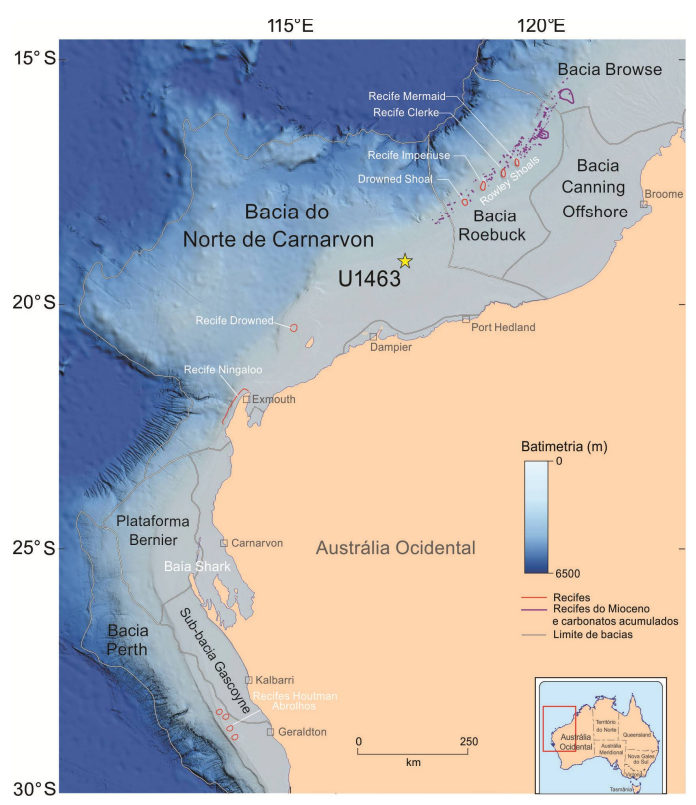

Figura 1: Localização do sítio U1463. Modificado de Gallagher et al. (2017). 
Para a escolha do sítio U1463C (18 57,9295'S, $117^{\circ}$ $\left.37,4336^{\prime} E\right)$ situado na porção externa da rampa carbonática (James et al. 2004), levou-se em consideração a atuação das monções e a Corrente de Leewuin como principais controladores da sedimentação na região, sob a influência do ITF, que afasta a corrente fria da região da costa noroeste australiana (Fig. 2). Esse sítio apresenta predominantemente sedimentos carbonáticos bioclásticos, areia e lama (James et al., 2004).

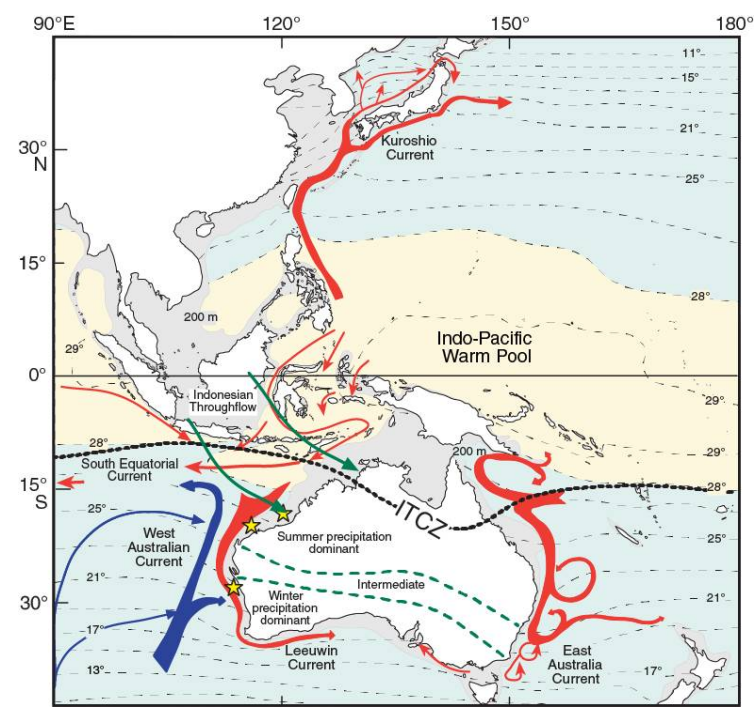

Figura 2: aspectos oceanográficos fundamentais que influenciam a NWS. Modificado de Gallagher et al. (2017).

\section{Métodos}

Sob as perspectivas da Expedição 356, a amostragem deste sítio teve o objetivo principal de se obter um registro carbonático tropical e subtropical do Plioceno ao Pleistioceno para determinar as taxas de subsidência, adquirir um registro interglacial das monções australiana e descrever a paleoceanografia da NWS.

Para o presente trabalho foram selecionados os dados de radiação gama natural (NGR - sigla em inglês) e dados susceptibilidade magnética (MS- sigla em inglês), obtidos à bordo do JOIDES Resolution através da ferramenta Triple Combo tool string, para a investigação de possíveis registros de quase-periodicidades. Tais medições permitem detectar emissão natural da radiação gama originada do decaimento dos radioisótopos U-238, Th232 e K-40, produzindo assim um registro da proveniência do arcabouço rochoso e a distribuição de fácies e transporte de sedimentos, e também, mede a susceptibilidade magnética do registro sedimentar que estão diretamente relacionados ao tipo suprimento detríticos contido no interior da bacia.

A análise das séries de dados NGR e MS, foram inicialmente interpolados e reamostrados em 0.1 metros e após passaram por um processo denominado "prewhitening" (falar o método que foi usado) e remoção da tendência. As análises espectrais das séries foiram estimadas a partir do método multitaper (MTM- sigla em inglês) relacionados a níveis de confianças estimados pelo modelo AR1 a partir scripts MATLAB classicredpad.m e classicconflevel.m (Kodama \& Hinnov, 2015). Com base no pacote de rotinas Astrochron (Meyers, 2014), foi possível avaliar as tendências para as frequências verificadas ao longo do registro estratigráfico a partir da análise harmônica evolutiva (EHA). Por fim, realizou-se a identificações de potencias componentes orbitais compreendidos pelo exame dos picos de frequência que correspondem à excentricidade, obliquidade e precessão para o intervalo de 3,2 - 5,2 Ma, baseado no modelo de Berger et. al. (1992).

\section{Resultados e Conclusões}

Os padrões obtidos via EHA (Fig. 3 - abaixo) indicam ligeira variabilidade nas taxas de sedimentação ao longo do intervalo, estimado em aproximadamente 1,7 Ma. O espectro de potências obtido por MTM (Fig. 3 - acima) são sugestivos para o registro das excentricidades longa e curta (comprimentos de onda: $\sim 45,5 \mathrm{~m}$ e $\sim 10 \mathrm{~m}$, aproximadamente).

$\mathrm{O}$ registro dos sinais de obliquidade $\left(\mathrm{O}_{1}\right.$ e $\mathrm{O}_{2}$ comprimentos de onda de $\sim 8,6 \mathrm{~m}$ e $\sim 5,0 \mathrm{~m}$ ) e de precessão $\left(\mathrm{P}_{1}\right.$ e $\mathrm{P}_{2}$ - comprimentos de onda de $\sim 3,4 \mathrm{~m} \mathrm{e}$

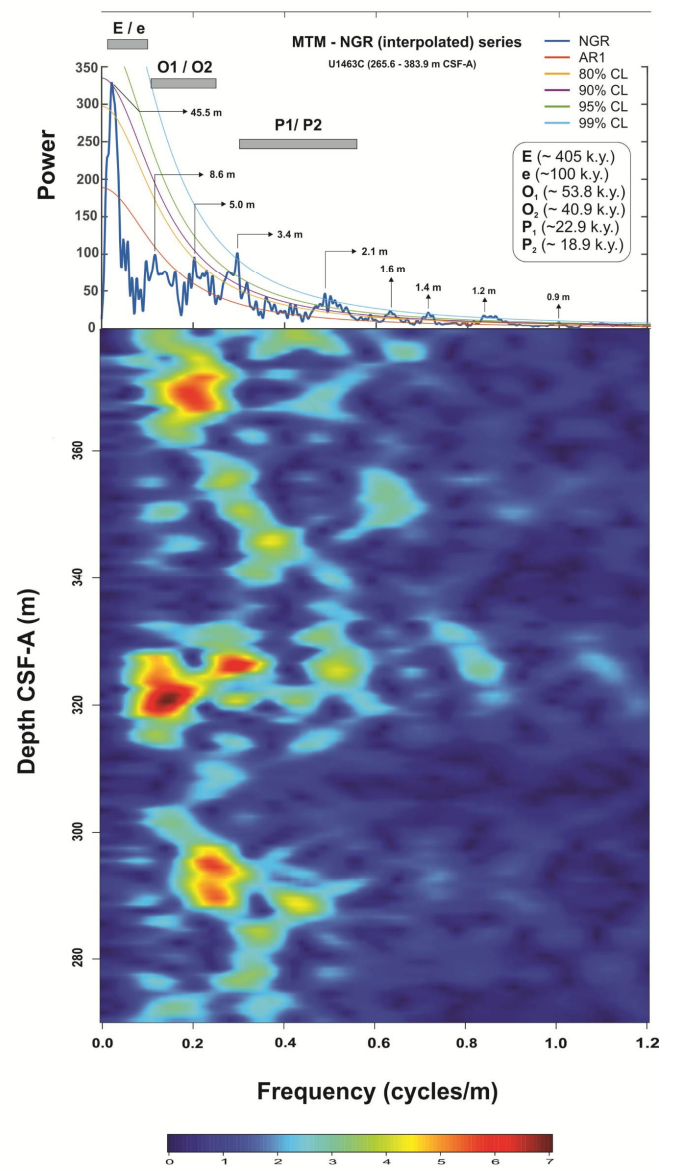

Figura 3: resultados dos espectros MTM (acima) e EHA (abaixo) para a série de dados NGR, após pre-whitening. 
2,1 m - vide Figura 4) puderam também ser sugeridos. $\mathrm{Na}$ próxima etapa deste estudo, buscaremos refinar os resultados através de análises de outras séries de proxies para este intervalo.
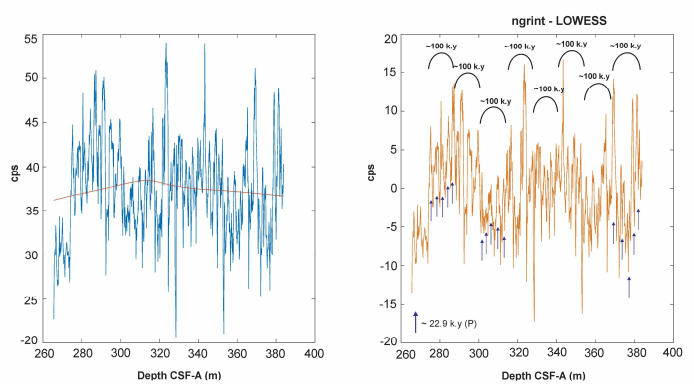

Figura 4: Esquerda - série de dados brutos de NGR após interpolação linear e reamostragem $(\mathrm{d}=0,1 \mathrm{~m}$; em azul), $\mathrm{e}$ associados à curva média ponderada a $60 \%$ (estimada pelo método Lowess; Cleveland (1979); em vermelho). Direita - curva de dados NGR (após remoção da curva Lowess a 60\%), com indicativos dos ciclos de excentricidade curta ( 100 k.y.) e de precessão ( 21 k.y.).

\section{Agradecimentos}

- Programa Institucional de Bolsas de Iniciação Cientifica (PIBIC/CNPq).

- Programas Estratégicos CAPES.

\section{Referências}

BERGER, A. LOUTRE , M.F., LASKAR, J., 1992. Stability of the astronomical frequencies over the Earth's History for Paleoclimate studies, Science 255, 560-556.

CLEVELAND, W.S., 1979. Robust locally weighted regression and smoothing scatterplots, Journal of the American Statistical Association 74 (368), 829-836,

GALLAGHER, S. J. et al. New frontiers in scientific drilling of the Indian ocean. Scientific Drilling, Copernicus $\mathrm{GmbH}$, v. 14, p. 60-63, 2012.

GALLAGHER, S. J. et al. Neogene history of the west pacific warm pool, kuroshio and leeuwin currents. Paleoceanography, Wiley Online Library, v. 24, n. 1, 2009.

GALLAGHER, S.J., FULTHORPE, C.S., BOGUS, K., and the Expedition 356 Scientists, 2017. Indonesian Throughflow. Proceedings of the International Ocean Discovery Program, 356: College Station, TX (International Ocean Discovery Program). http://dx.doi.org/10.14379/iodp.proc.356.2017.

GARIDEL-THORON, T. de et al. Stable sea surface temperatures in the western pacific warm pool over the past 1.75 million years. Nature, Nature Publishing Group, v. 433, n. 7023, p. 294, 2005.
JAMES, N. P., BONE, Y., KYSER, T. K., et al., 2004, The importance of changingoceanography in controlling late Quaternary carbonate sedimentation on a high-energy, tropical, oceanic ramp: northwestern Australia, Sedimentology 51 (6), 1179-1205.

KODAMA , K.P, HINNOV, L.A., 2015. Rock Magnetic Cyclostratigraphy, New Analytical Methods in Earth and Environmental Science Series. Wiley-Blackwell, New York, $176 \mathrm{pp}$.

MEYERS, S.R., 2014. Astrochron: An R Package for Astrochronology (available at http://cran.rproject.org/package=astrochron).

VEEVERS, J.; POWELL, C. M.; ROOTS, S. Review of seafloor spreading around australia. i. synthesis of the patterns of spreading. Australian journal of earth sciences, Taylor \& Francis, v. 38, n. 4, p. 373-389, 1991. 